\title{
Online beam monitoring on targets at an ion beamline by infrared thermography
}

\author{
by M. Tomut*, C. Hubert**, K.Kupka**, D. Severin, M. Bender and C.Trautmann** \\ *GSI Helmholzzentrum für Schwerionenforschung, Planckstr.1, 64291 Darmstadt, Germany, m.tomut@gsi.de \\ **TU Darmstadt, Darmstadt, Germany
}

\begin{abstract}
First results of using thermography for profile monitoring of high intensity pulsed ion beams on thin graphite targets are presented. IR measurements of time dependent evolution of beam-induced target temperature were used as input in finite element calculations of thermal stress wave amplitudes in targets. This technique shows a promising potential as monitoring method in high-power accelerator facilities. For beam diagnostic, thermographic screens made out of carbon materials would be a cheaper alternative to luminescence screens.
\end{abstract}

\section{Introduction}

The thermal print of an ion beam on a target is related to the power density deposited in the target [1]. Infrared radiometry is one of the few methods suitable for the monitoring of high-intensity ion beams on targets. This work summarizes first results on online infrared imaging of beam spot size on thin graphite targets in experiments with ion beams at MeV-GeV energies using a FLIR SC 7500 high sensitivity, cooled IR camera. Due to their good thermomechanical properties and high emissivity, graphite targets are ideal for thermography-based beam diagnostic.

\section{Results and discussion}

Figure 1 presents the time evolution of the temperature maximum within the beam spot of 3.6 MeV/u Au ions. The high sensitivity and low integration time of the camera provide a high accuracy monitoring of intensity variation during individual pulses. This type of information was used as input in finite element calculations of beam-induced thermal stress in targets. About $2 \mathrm{~s}$ are necessary for the temperature in the target to reach the steady state at the beginning of the irradiation, for the given beam parameters. Upon the beam stop, the time constant of the temperature decay in the target can be related to the thermal conductivity of the target materials.

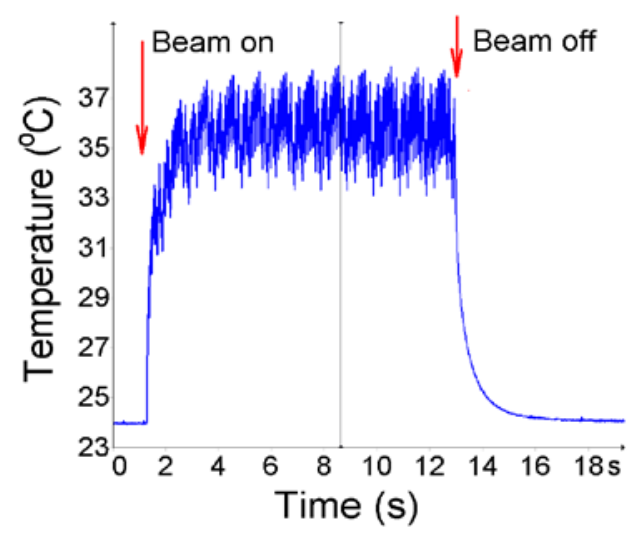

Figure 1: Temporal evolution of temperature within the center of the beam spot during irradiation with $1 \times 10^{9}$ $\mathrm{i} / \mathrm{cm}^{2} \mathrm{~s}{ }^{197} \mathrm{Au}$ ions with a energy of $3.6 \mathrm{MeV} / \mathrm{u}$, $4 \mathrm{~ms}$ pulse length and a repetition rate of $38 \mathrm{~Hz}$. 


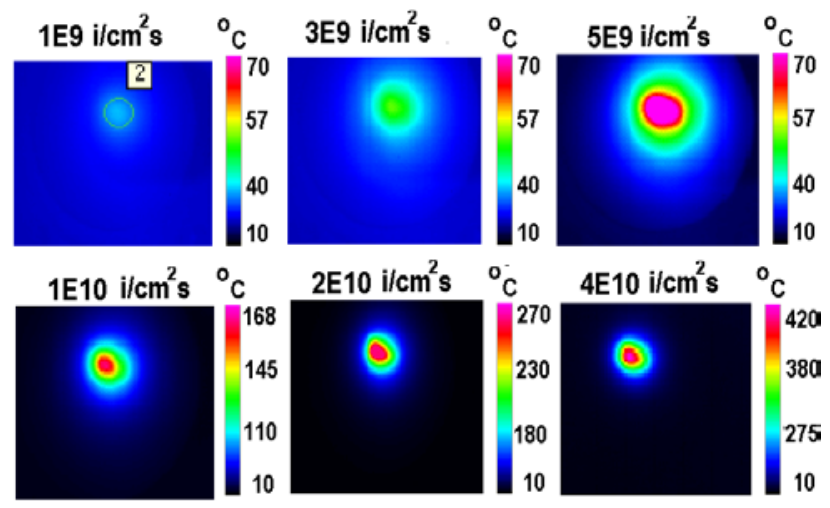

Figure 2: Sequence of infrared images of beam spot on thin graphite targets with increasing beam flux. Each image corresponds to maximum intensity during a pulse, for a $3.6 \mathrm{MeV} / \mathrm{u}, 4 \mathrm{~ms}, 38 \mathrm{~Hz}{ }^{197} \mathrm{Au}$ beam.

To get accurate temperature distribution from the radiometric image of the ion heated target, previous calibration of the emissivity of the target and environment and of the transmittance of the beamline IR viewport are necessary. Figure 2 shows a series of thermal prints of Au ion beam pulses with increasing intensity on graphite targets. Snapshots are taken after the temperature in the targets reached a steady state condition ( $>2 \mathrm{~s}$ ) and at the maximum pulse intensity. The increase of the maximum temperature in the beam spot with increasing ion flux is shown in Figure 3. A detailed analysis of the fully radiometric image is used for inferring the beam power density profiles providing the beam intensity profile. To validate these results, cross-checks using luminescence targets are done. This beam diagnostic technique is particularly suited for high intensity cases where other techniques would fail.

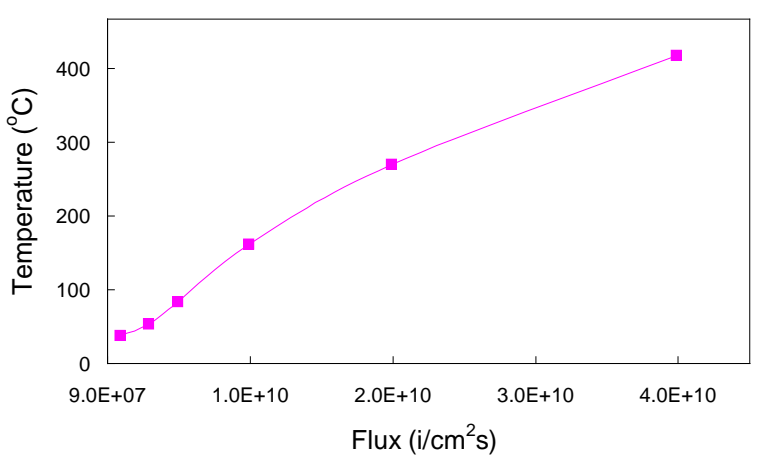

Figure 3: Maximum temperature recorded within the beam spot on thin graphite targets exposed to 3.6 MeV/u ${ }^{197} \mathrm{Au}$ ions, with increasing beam intensity, as a function of beam flux.

\section{REFERENCES}

[1] H. Buttig, "Beam Diagnostic by Infrared Thermography" ZKF-453 Report Forschungszentrum Rossendorf, Aug 1981. 